\title{
Final Report: Biomolecular simulation using Amber and CHARMM
}

(DE-FG03-01ER25496), for project period 9/1/01 through 8/31/04.

\section{Principal Investigators:}

David A. Case, Professor

Dept. of Molecular Biology

The Scripps Research Institute

10550 N. Torrey Pines Rd.

La Jolla, CA 92037

phone: 858-784-9768

e-mail: case@ scripps.edu
Charles L. Brooks, III, Professor

Dept. of Molecular Biology

The Scripps Research Institute

10550 N. Torrey Pines Rd.

La Jolla, CA 92037

phone: 858-784-8035

e-mail: brooks@scripps.edu

\section{Technical progress during the the project}

This project supports the development of software using terascale computers to carry out molecular simulations of protein function and macromolecular interactions. We are building on the existing CHARMM and Amber simulation packages, adapting them in novel ways to massively parallel architectures and high-performance CPUs. Three principal avenues being pursued are:

[a] Improvements in load-balancing and communication for large-scale particle-mesh Ewald (PME) simulations of solvated biomolecules.

[b] Modern techniques for accelerating convergence of sampling of configuration space offer promise for further exploitation of massively parallel architectures. These methods include parallel tempering and "lambda dynamics" procedures that connect multiple, synchronized results from PME simulations like those described in part [a].

[c] The implementation of efficient and scalable algorithms that move towards lower-resolution models in ways that can be carefully calibrated against atomic-level solvated simulations.

\subsection{Progress report overview}

This project focused on critical performance issues in Amber and CHARMM. This was a key objective of the funding from DOE. Our work has centered around: 1) single processor profiling and performance analysis to identify routines to target for optimization in both CHARMM and Amber; 2) improvement of single processor performance on multiple platforms for target routines; 3 ) detailed exploration of approaches to enhance the parallel scalability of both CHARMM and Amber.

The focus of performance on parallel platforms was also an area where we spent a lot of time. In particular, in Amber we have undertaken a major re-write to use spatial decomposition to improve parallel performance. This effort is now nearly complete, and some details are reported below. These efforts have been successful enough that there was both an "interim" release of a new parallel code (done by Bob Duke at UNC-Chapel Hill) in the summer of 2003, and a full incorporation of these ideas in the Amber8 release in March 2004.

Some details of particular aspects of the optimization work are given in the next few sections. 


\subsection{Overall speedups for CHARMM and Amber}

The steps outlined above only describe the most important aspects of code tuning carried out during the project. Overall, our efforts have been gratifying in terms of general improvements in efficiency. The following tables summarizes progress for our main protein+water test case, on various machines widely used for current generation supercomputing.

\begin{tabular}{|cccc|}
\hline \multicolumn{4}{|c|}{ Timings at Blue Horizon (SDSC) } \\
\hline nproc & amber7 & $c 29 a 1$ & namd2.4 \\
\hline 1 & 2538 & 2643 & 2986 \\
2 & 1229 & 1371 & 1809 \\
4 & 658 & 724 & 930 \\
8 & 375 & 414 & 516 \\
16 & 228 & 255 & 308 \\
32 & 162 & 195 & 202 \\
64 & 124 & 182 & 124 \\
\hline
\end{tabular}

\begin{tabular}{|cccccc|}
\hline \multicolumn{7}{c|}{ Timings on SGI Origin } \\
nproc & amber6 & amber7 & $c 29 a 1$ & namd2.4 & namd2.5b1 \\
\hline 1 & 1797 & 1397 & 1543 & 2320 & 2403 \\
2 & 960 & 687 & 817 & 1259 & 1296 \\
4 & 508 & 374 & 436 & 625 & 648 \\
8 & 280 & 206 & 244 & 312 & 325 \\
16 & 174 & 127 & 149 & 163 & 166 \\
32 & 120 & 93 & 115 & 87 & 90 \\
64 & 97 & 78 & 114 & 52 & 54 \\
\hline
\end{tabular}

We are continuing to make good progress in this area: for example, the amber7 time of $93 \mathrm{sec}-$ onds at 32 processors for the above benchmark is now down to 57 seconds (a $63 \%$ increase in speed) as a result of work described in the next section.

\subsection{Moving from replicated data to spatial decomposition}

All previous parallel versions of Amber have used a replicated data model, in which all processors have access to the coordinates of all atoms. This makes it easy to implement new force fields, but limits parallel scaling. We are moving now towards a spatial decomposition model.

The accompanying table shows an analysis of message sizes for our current spatial decomposition scheme, compared to the current released version. (The final line shows a simple change that is currently being tested.)

The upshot is that communication costs on 64 nodes have been reduced by about a factor of 4 , compared to the current code. This is especially important on systems with slower communications (say gigabit Ethernet), but is significant on even the most sophisticated clusters. 


\begin{tabular}{|cccccc|}
\hline \multicolumn{6}{|c|}{ Message sizes for protein + water (140,000 atoms) } \\
\hline Code & \#proc & FFT & Coordinates & Forces & Total \\
\hline Amber7 & 2 & 1.607 & 0.423 & 0.423 & 2.454 \\
& 4 & 1.205 & 0.635 & 0.635 & 2.475 \\
& 8 & 0.714 & 0.741 & 0.741 & 2.196 \\
& 16 & 0.395 & 0.793 & 0.793 & 1.982 \\
& 32 & 0.218 & 0.820 & 0.820 & 1.859 \\
& 64 & 0.111 & 0.834 & 0.834 & 1.779 \\
\hline Amber8 & 2 & 1.607 & 0.206 & 0.114 & 1.928 \\
& 4 & 1.205 & 0.262 & 0.114 & 1.582 \\
& 8 & 0.714 & 0.293 & 0.118 & 1.126 \\
& 16 & 0.394 & 0.307 & 0.135 & 0.838 \\
& 32 & 0.218 & 0.316 & 0.171 & 0.706 \\
& 64 & 0.111 & 0.314 & 0.170 & 0.595 \\
& 64 & 0.111 & $0.170 *$ & 0.170 & 0.451 \\
\hline
\end{tabular}

Values are in millions of double-precision words per step. Asterisk indicates a code modification nearly but not completely finished. Hence, a spatial decomposition code has been implemented in Amber which reduces communication costs (the main parallel bottleneck) by factors of 4 or more. Performance on large numbers of processors is currently being profiled and optimized.

\subsection{More recent work on spatial decomposition}

The spatial-decomposition approach to parallel molecular dynamics (MD) has improved parallel performance in AMBER, especially in scaling to many more processors. The traditional parallel method in AMBER is the replicated-data method. The greatest impediment to parallel scaling is the all-to-all force and coordinate communications at each step. We have reduced the communications by an order of magnitude in those communications.

Our approach to improving scaling in sander, the MD module of AMBER, is guided by a philosophy of non-invasiveness and full functionality. This philosophy was adopted to keep the existing code as unchanged as possible so that other developers would not be impeded or discouraged from continuing to develop new methodologies. However, this philosophy has been the source of some very troublesome and unanticipated problems for the spatial-parallel implementation. We have tested several approaches to the problems imposed by the philosophy and spatial decomposition and come up with workable solutions at the expense of several unacceptable solutions. The unacceptable solutions, though they did work, compromised the intended goal of fast and simple code by being either far too expensive computationally, or were far too complex, fragile, and very difficult to debug and maintain.

The current implementation of spatial-decomposition parallel sander has capabilities of constant temperature and pressure, constraints on bonds to hydrogen using SHAKE, extra points or lone pairs for water models: TIP4P and TIP5P, and polarization using dipoles.

The method has been improved significantly this year by changing the algorithm for assigning atoms to processors so that assignment is by heavy atom only, and hydrogens stay with the heavy atom to which they are bonded. The change eliminates the necessity of checking and communicating atoms which are involved in bond constraints but owned by another processor. The price of using a slightly larger cutoff is far lower than the price of the bookeeping and communications involved in 
the original method.

\section{Factor IX 90,000 atoms}

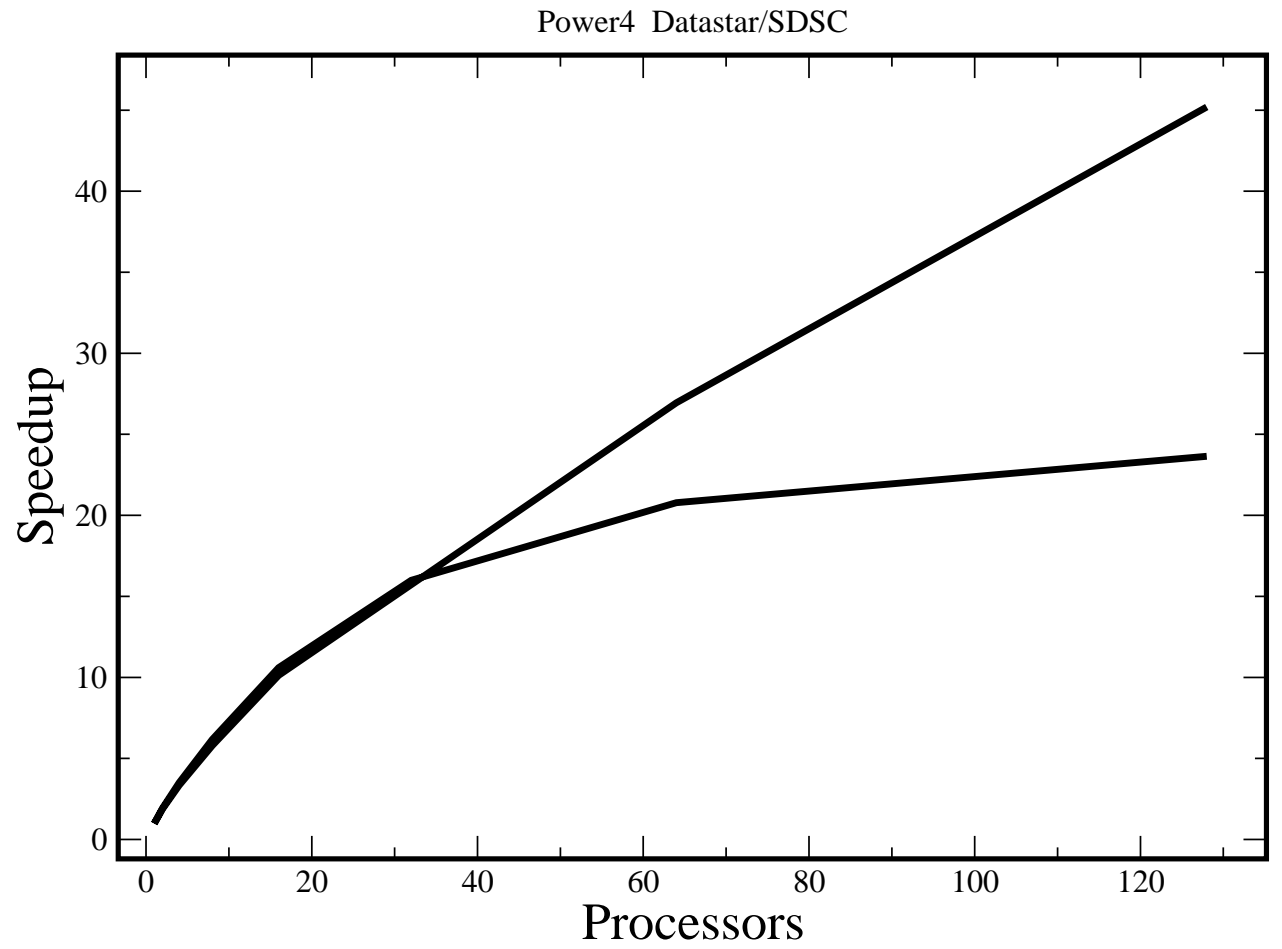

Representative scaling for sander and psander are shown above. The lower curves are for replicated data method, higher are for spatial decomposition. The speedup is defined as the time for one processor run divided by the time for the multiprocessor run. As expected, both sander and psander have the same times for 1 processor. The speedup bottleneck is now the transpose communications in the 3D-FFT and is the current target of effort. Our new compact decomposition and processor groupings are addressing the problem with the current implementation.

Component communications times and sizes are shown below for replicated data and spatial decomposition. 


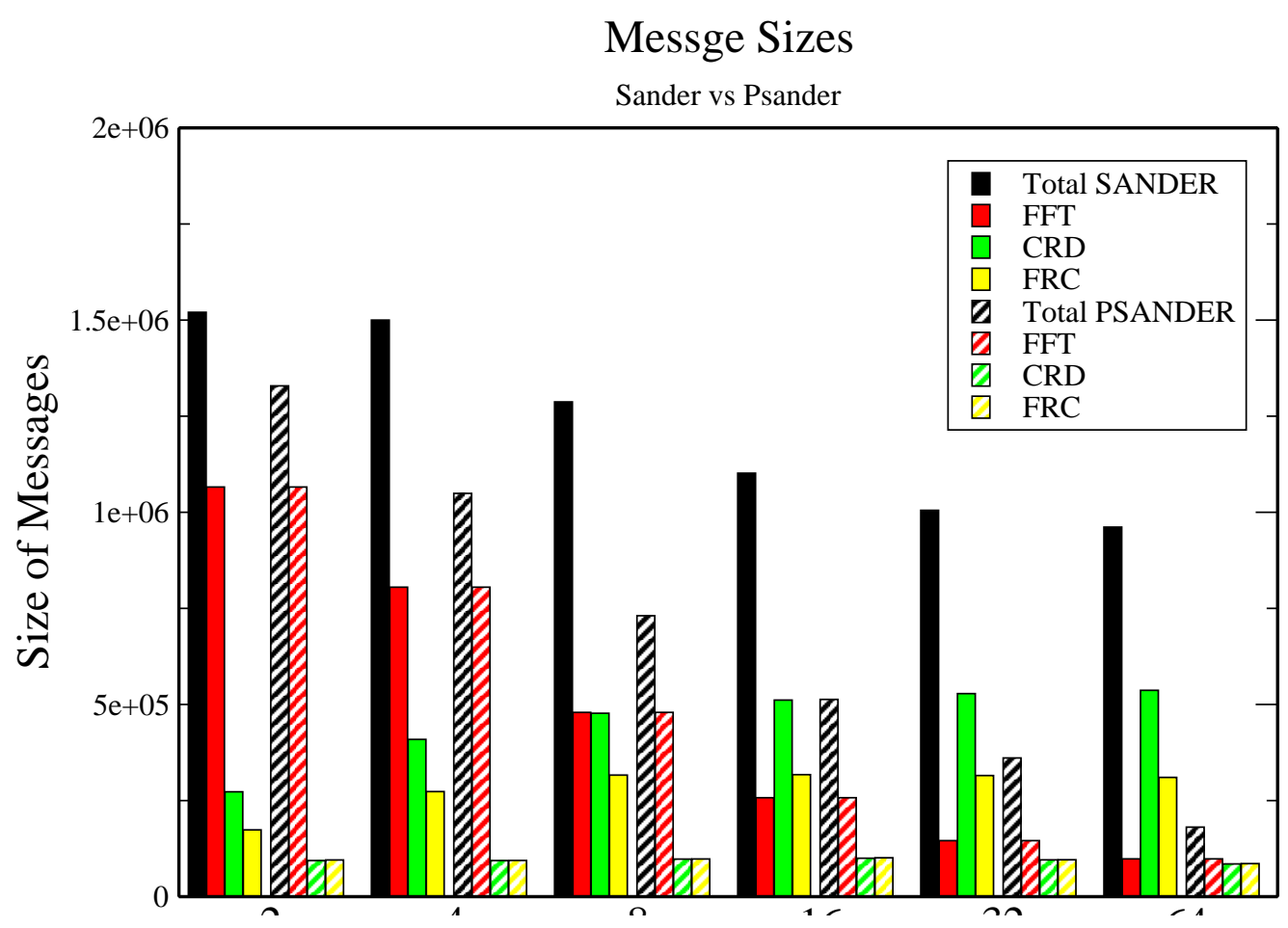

AMBER: Component percent timings
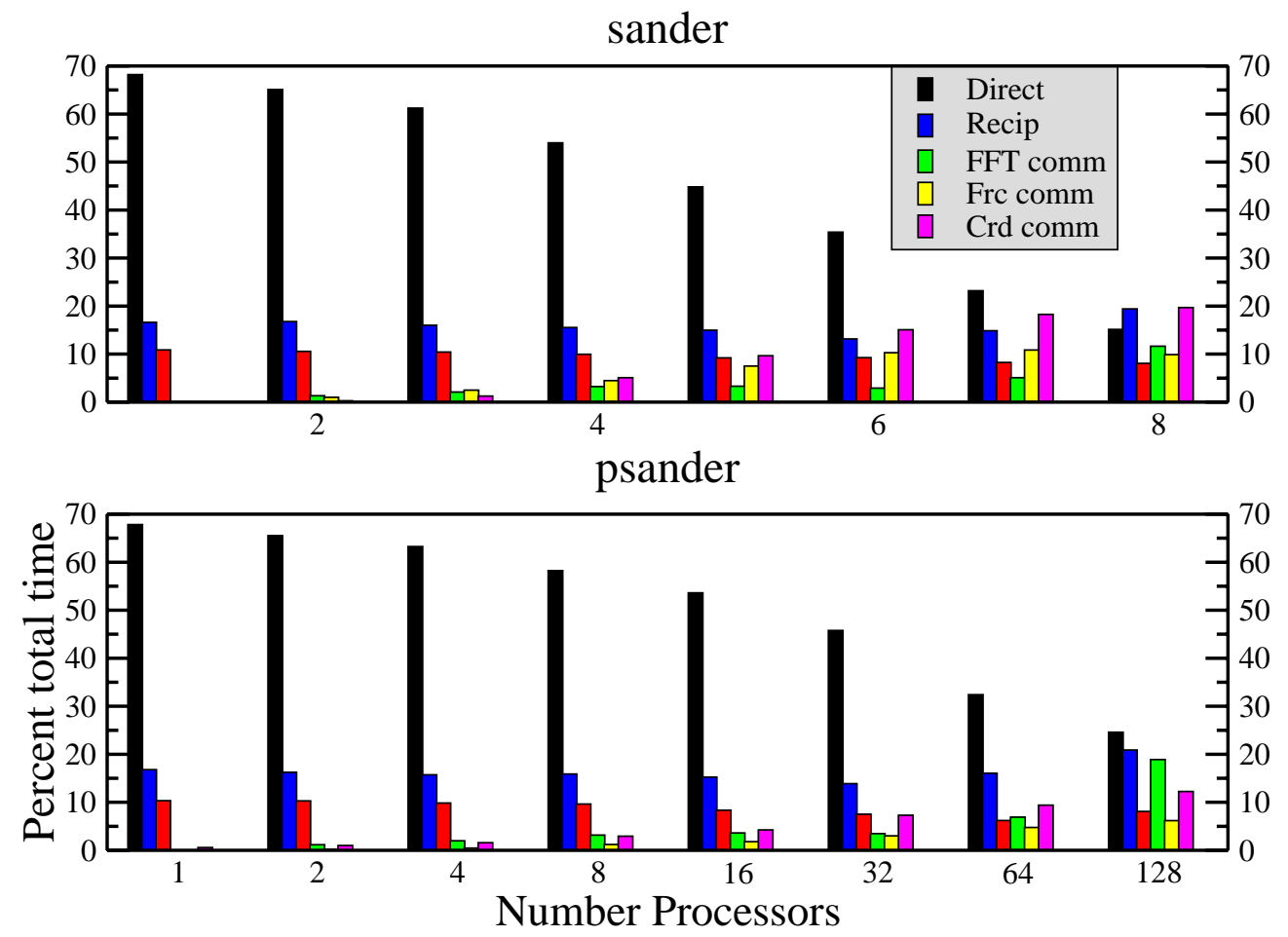

Current efforts are targeted at performance improvements in the bookeeping part of the spatial decomposition algorithm, and in more compact and flexible spatial decompositions. We are 
investigating methods of including lone pairs, or extra points, for the non-water atoms. That problem is complicated by the algorithm which includes nearby heavy atom positions in the determination of lone pair positions, atoms which may not be known by a processor which owns the lone pairs.

\subsection{Optimizations for Intel architectures}

In molecular dynamics simulations the calculations of the nonbonded interactions are a computational bottleneck. These interactions depend upon the interparticle distances. On Intel 32 bit architectures (IA32) the fastest methods to evaluate reciprocal square roots utilize the Streaming SIMD (Single-instruction multiple-data) Extensions for double precision (SSE2) operations. Tuning the AMBER or CHARMM source codes to enable automatic vectorization, i.e., generation of SSE2 instructions by compilers, introduces memory cache penalties as well as additional loop overheads. Ironically, the cache penalties stem from the loss of spatial locality due to the SSE2 requirement of contiguous memory operands.

For CHARMM the gain from vectorization does not compensate the cache and loop losses. Significant rewriting of the van der Waals computation, which is more flexible and thus complicated in CHARMM than in AMBER, probably would produce a small performance improvement, a couple of percent, for CHARMM on IA32 platforms. That code would be made architecture specific to avoid larger performance degradation on workstations, such as SGI machines. On those platforms the tuning for SSE2 conflicts with existing optimizations to enhance software pipelining. Consequently, because of the dim prospects for improvement of CHARMM and the concomitant software complexity, IA32 SSE2 tuning will not be included in CHARMM releases at the present time. On IA32 platforms the SSE2 tuned AMBER is approximately eight percent faster than the original AMBER as measured by execution times of a typical explicit solvent protein simulation. On SGI platforms the SSE2 tuned version is approximately four percent slower.

It is increasingly important that codes run well on Intel and Athlon chips, since these are becoming the work-horses of scientific computation. Parallel performance is important here as well, but our initial steps have looked at compiler and code optimizations. The accompanying table gives results for a implicit solvent model, with 18,000 atoms, in a generalized Born simulation of 48 steps. Timings are given for a $2.0 \mathrm{Ghz}$ Pentium IV, single processor.

\begin{tabular}{|clc|}
\hline version & \multicolumn{1}{c}{ compiler } & time,sec \\
\hline Amber 7 & g77-3.1.1 & 806 \\
& ifc 7 & 526 \\
Amber 8 & g77-3.1.1 & 601 \\
& ifc 7 & 316 \\
& ifc 7 + MKL $^{\mathrm{a}}$ & 288 \\
& & $187^{\mathrm{b}}$ \\
\hline
\end{tabular}

(a) Using Intel's Math Kernel Library

(b) multiple-time-step option; slight degradation of energy conservation

The results of these optimizations show that Amber 8 is about twice as fast as Amber 7, and with a very modest algorithm change, (that has no noticeable effects on energies,) Amber 8 is about $3 x$ Amber 7. These advancements will significantly improve the attractiveness of implicit solvent models for the larger systems we are pointing toward in the future. 


\section{Ongoing projects}

Many of the tasks outlined above (especially regarding spatial decomposition) are nearly complete, and should be finished by mid-summer. A key point for the next year to integrate the parallel extensions (begun in Amber) to CHARMM; we expect this to be completed in the summer/fall time frame. In addition, multiple copy replica-exchange methods (not described in detail here) are being finalized, and will be distributed as a part of the Multiscale Modeling Tools suite (see http://mmtsb.scripps.edu). One major additional on-going project is outlined in the following paragraphs.

\subsection{Hybrid PME-Ewald methods}

The most time-consuming part of almost any biomolecular simulation consists of determining the energies and forces arising from non-bonded interactions. Generally, interactions must be considered out to quite large distances to avoid artifacts that would otherwise arise from neglect of longrange interactions, but the number of such interactions grows quickly as the cutoff distance increases. For periodic systems, it is possible to circumvent this problem to a large extent, by splitting the interaction operator (in this case, the Coulomb interaction) into two pieces. The first is short-ranged, and can be handled by conventional methods, with a cutoff of 8 or $9 \AA$; the second piece is longer ranged, but is rapidly convergent in reciprocal (Fourier) space. If the molecular charge distribution can be represented with a grid, the reciprocal term can be computed by discrete Fourier transforms, handled by fast Fourier (FFT) algorithms. As mentioned above, this particle-mesh-Ewald (PME) approach is now the method of choice, for both accuracy and performance considerations.

Although the computation cost to prepare the reciprocal space charge grid is not negligible, the quality of parallel implementations of PME primarily depend upon methods for efficiently handling three-dimensional FFT's. There are a variety of approaches to this, but none scales very well, particularly for the relatively small grids (50 to 100 points on a side) of use in biomolecular simulations.

Several workers have noted that a hybrid Ewald-PME scheme might help get past this bottleneck, but (as far as we know) there is no generally available code to do this. A "hybrid" scheme uses the PME approach for two dimensions of the reciprocal sum, but uses an Ewald sum over reciprocal vectors for the third dimension. For many cases, this means that each processor can perform its fraction of the two-dimensional FFT's that are needed, working entirely in local memory; the sum along the third dimension can also be distributed among processors, so that the total effort can be well load-balanced and not require any communications. We emphasize that we have not yet implemented this model; however, since code for both "regular" and PME variants of the Ewald method are in the programs, we do not expect significant difficulties. The performance penalty for one processor, compared to current PME models, should be small, and this may be overcome by better scaling once the processor numbers increase. 